\title{
Intervenções de fisioterapia respiratória para crianças e adolescentes com fibrose cística: uma revisão integrativa
}

\author{
Respiratory physiotherapy interventions for children and adolescents with cystic fibrosis: \\ an integrative review
}

VALANDRO, Amanda Franciele ${ }^{1}$; HEINZMANN-FILHO, João Paulo ${ }^{1,2}$.

\begin{abstract}
Resumo
Introdução: A fibrose cística é uma doença multissistêmica, sendo as complicações respiratórias, as principais responsáveis pela morbimortalidade da doença. $\mathrm{O}$ tratamento envolve equipe multidisciplinar, no qual, a fisioterapia respiratória tem como objetivo otimizar a função pulmonar. Diversas intervenções de fisioterapia respiratória (convencionais, baseadas em volume, instrumentais e exercícios ventilatórios) são utilizadas no tratamento da fibrose cística, e mapear os estudos relacionados a elas contribui no manejo da doença. Objetivo: Revisar as intervenções fisioterapêuticas apresentadas, na literatura indicada, para remoção de secreção das vias aéreas de crianças e adolescentes com fibrose cística. Métodos: Trata-se de uma revisão integrativa, realizada através do Pubmed, LILACS, SciELO, Cochrane e PEDro. Utilizou-se a seguinte estratégia de busca: cystic fibrosis AND physical therapy modalities OR bronchial hygiene. Foram incluídos estudos que utilizaram intervenções fisioterapêuticas para a remoção de secreção das vias aéreas de crianças e adolescentes com fibrose cística. Resultados: De um total de 1.526 artigos, apenas 22 foram incluídos. Os artigos totalizaram 708 participantes, com idades oscilando entre três semanas e 18 anos. As intervenções fisioterapêuticas utilizadas apresentaram variabilidade, sendo, as mais frequentes, as convencionais (drenagem postural, percussão, vibração), em 16/22 estudos, seguidos das instrumentais (dispositivo/máscara de pressão expiratória positiva e Flutter ${ }^{\circledR}$ ), em 12/22, dos exercícios ventilatórios (7/22) e das baseadas em volume (ciclo ativo da respiração, drenagem autogênica) em 6/22 artigos. Além disso, conforme ocorre um aumento da faixa etária, há uma redução por intervenções fisioterapêuticas exclusivamente convencionais. Conclusão: Houve grande variabilidade nas intervenções de fisioterapia respiratória utilizadas para a remoção de secreção das vias aéreas nesse mapeamento realizado em crianças e adolescentes com fibrose cística. No entanto, evidenciou-se certo predomínio de intervenções convencionais. A escolha da terapêutica parece ter sido influenciada pela idade dos participantes.
\end{abstract}

Palavras-chave: Fibrose Cística; Modalidades de Fisioterapia; Higiene Brônquica; Pediatria.

\footnotetext{
${ }^{1}$ Centro Universitário Cenecista de Osório (UNICNEC), Osório-RS, Brasil.

${ }^{2}$ Email: joaopauloheinzmann@hotmail.com
} 


\section{Abstract}

Introduction: Cystic fibrosis is a multisystemic disease with respiratory complications the main factor responsible for the morbidity and mortality caused by the disease. The treatment requires a multidisciplinary team, in which chest physiotherapy aims to optimize pulmonary function. Several respiratory physiotherapy interventions (conventional, volume-based, instrumental and ventilatory exercises) are used in the treatment of cystic fibrosis. To review the available evidence of these techniques helps to improve the management of the disease. Objective: To review physical therapy interventions of airway clearance for children and adolescents with cystic fibrosis. Methods: It is an integrative review, carried out via Pubmed, LILACS, SciELO, Cochrane and PEDro. The following search strategy was used: cystic fibrosis AND physical therapy modalities OR bronchial hygiene. Studies that used physical therapy interventions to remove airway secretion in children and adolescents with cystic fibrosis were included. Results: In a total of 1.526 articles, only 22 were included. The articles totalled 708 participants, with their ages ranging from three weeks to 18 years. There was a large variability of physical therapy interventions with conventional techniques (postural drainage, percussion, vibration) the most frequent ones (16 studies). The second most prevalent techniques (12 studies) were instrumental (positive expiratory pressure device/mask and Flutter ${ }^{\circledR}$ ), followed by ventilatory exercises (7 studies) and volume-based exercises (active breathing cycle, autogenic drainage) in 6 studies. Additionally, with the increase of age, fewer interventions using exclusively conventional techniques tend to be applied. Conclusion: There was considerable variability in respiratory physiotherapy interventions used to remove secretions from the airways in this review performed in children and adolescents with cystic fibrosis. However, there was a predominance of conventional interventions. The choice of therapy seems to have been influenced by the age of the participants.

Keywords: Cystic Fibrosis; Physical Therapy Modalities; Bronchial Hygiene; Paediatrics.

\section{Introdução}

A fibrose cística (FC) é uma doença genética, causada pela disfunção do gene da proteína cystic fibrosis transmembrane conductance regulator (CFTR) responsável por regular a condutividade do cloro ${ }^{1}$. É considerada uma doença multissistêmica, afetando os locais em que a CFTR atua, como as glândulas sudoríparas, os pulmões, o pâncreas e o sistema reprodutor ${ }^{1,2}$. Manifestandose principalmente pelo suor salgado, pela tosse produtiva e persistente, pelo baixo crescimento ou ganho de peso, pelas fezes gordurosas e por infecções pulmonares recorrentes ${ }^{3,4}$.

Estima-se que a incidência da FC, no Brasil, seja de 1/7.576 nascidos vivos, com predomínio de ocorrência em descendentes de caucasianos, havendo mais registros nos estados da Região Sul ${ }^{4}$. A expectativa de vida desses pacientes vem crescendo $^{5}$, sendo que, nos dias atuais, estima-se uma sobrevida acima dos 40 anos $^{6}$. Essa conquista vem sendo atribuída aos avanços do diagnóstico, do tratamento e devido ao aumento de centros de referência ${ }^{1,4}$.

O tratamento da doença aborda o atendimento multidisciplinar, utilizando recursos medicamentosos (antibioticoterapia, broncodilatadores, agentes anti-inflamatórios), reposição enzimática, suporte nutricional, prática de atividade física e a fisioterapia respiratória (FR ${ }^{2,3}$. Devido às implicações respiratórias serem responsáveis por piorar o cenário da doença, otimizar a função pulmonar é o maior objetivo da $\mathrm{FR}^{7}$. Dados relatam que a FR é essencial, considerando a produção diária e espessa de muco e os quadros recorrentes de infecções pulmonares ${ }^{7,8}$. Isso acarreta obstrução crônica das vias aéreas, ocasionando exacerbações e hospitalizações ${ }^{9}$. De forma progressiva, pode ocorrer, também, a redução das atividades de vida diária e a tolerância ao exercício físico ${ }^{10}$.

Atualmente, a FR conta com diversas intervenções para o tratamento de crianças e adolescentes 
com $\mathrm{FC}^{9,10}$, visando desobstruir as vias aéreas, aperfeiçoar a relação ventilação-perfusão, reduzir a resistência pulmonar, bem como melhorar o condicionamento físico e a qualidade de vida ${ }^{10}$. Nestes procedimentos, estão inclusas as intervenções convencionais, baseadas em volume instrumentais, $\mathrm{e}$ os exercícios ventilatórios ${ }^{9,10}$, que, normalmente, são usados em conjunto com terapias de inalação, para facilitar a remoção do muco ${ }^{1}$. Dentre as intervenções fisioterapêuticas convencionais, podem-se citar, por exemplo, a drenagem postural, a percussão torácica e a vibração. Nas baseadas em volume, o ciclo ativo da respiração, a drenagem autogênica, drenagem autogênica modificada ou assistida podem ser listadas. Nas intervenções instrumentais, destacam-se as pressões positivas oscilatórias ou resistores de fluxo ${ }^{7,9,10}$. Além do mais, nos exercícios ventilatórios, podem ser inclusos a respiração diafragmática, os exercícios expiratórios forçados, profundos e prolongados ${ }^{8}$.

A diretriz nacional mais recente de diagnóstico e tratamento da FC sugere, para a prática da FR, que sejam aplicadas intervenções instrumentais, tais como a máscara de pressão expiratória positiva e os dispositivos de pressão oscilatória positiva do tipo Flutter ${ }^{\circledR}$, Shaker $^{\circledR}$ e Acapella ${ }^{\circledR}$, devido à independência e preferência do paciente ${ }^{4}$. No entanto, não foram citadas as terapias mais indicadas para o tratamento dos pacientes dependentes, como recém-nascidos e lactentes. Além disso, embora existam diretrizes e guias ${ }^{4,10}$,que explanem e/ou recomendem as intervenções fisioterapêuticas para essa população, não há detalhes quanto à estratégia de busca utilizada para a seleção dos artigos incluídos nos documentos.

Até o presente momento, ocorreram poucas revisões integrativas brasileiras que mapeassem as intervenções de FR, visando à remoção de secreção das vias aéreas de crianças e adolescentes com FC, justificando o desenvolvimento deste manuscrito. Dessa forma, o objetivo deste estudo foi revisar as intervenções fisioterapêuticas apresentadas na literatura indicada para essa finalidade.

\section{Métodos}

Trata-se de uma revisão integrativa, realizada por meio de pesquisa nas bases de dados PubMed, Scientific Electronic Library Online (SciELO), Literatura Latino-Americana e do Caribe em Ciências da Saúde (LILACS), Cochrane Library e Physiotherapy Evidence Database (PEDro).

Foram selecionados, estudos observacionais e de intervenção, sem filtros quanto ao idioma e ao ano de publicação. A seleção dos artigos ocorreu em março e abril de 2019. A busca utilizada para a seleção dos artigos foi baseada em três palavras-chave, associadas com descritores booleanos, com a seguinte estratégia: cystic fibrosis AND physical therapy modalities OR bronchial hygiene. Esses descritores deveriam constar, pelo menos, no título, no resumo ou nas palavras-chave.

Foram incluídos, estudos com crianças e adolescentes, desde o nascimento até 18 anos de vida $^{11}$, com diagnóstico de FC (de acordo com os critérios de cada autor), e submetidas ao tratamento com FR, para a remoção de secreção das vias aéreas (conceituada, através da função pulmonar e/ ou do volume de escarro e/ou da análise de expectoração). Em contrapartida, foram excluídos, artigos sem relação com o tema, estudos com amostras adultas, artigos sem livre acesso, estudos de revisão e relatos de casos. Também, foram excluídas pesquisas que abordavam somente a intervenção fisioterapêutica durante o exercício ou para o treinamento muscular respiratório.

Todos os artigos encontrados passaram pela leitura dos títulos e/ou leitura dos resumos, para avaliar a adequação quanto aos critérios de elegibilidade. Os estudos que apresentaram os critérios predeterminados tiveram o texto completo adquirido para análise e extração dos dados. A busca e a 
análise dos artigos foram conduzidas de forma independente, por dois avaliadores, sendo qualquer divergência resolvida por consenso.

Registraram-se, as seguintes características dos estudos: nome do primeiro autor, ano de publicação do estudo, país (origem) da coleta de dados, delineamento do estudo, faixa etária, tamanho amostral, função pulmonar (espirometria - volume expiratório forçado em um segundo $\left[\mathrm{VEF}_{1}\right] \mathrm{e}$ capacidade vital forçada $[\mathrm{CVF}]$ ) e as intervenções de FR utilizadas para a remoção de secreção das vias aéreas.

As intervenções fisioterapêuticas foram agrupadas e classificadas de acordo com quatro grupos, de forma adaptada ${ }^{8}$ :

1) Convencionais: drenagem postural, percussão torácica, vibração/vibrocompressão e técnica de expiração manual passiva;

2) Baseadas em volume ciclo ativo da respiração, drenagem autógena, drenagem autógena modificada ou assistida e expiração lenta total com a glote aberta;

3) Instrumentais: dispositivo/sistema de pressão expiratória positiva (PEP), Flutter ${ }^{\circledR}$, Shaker $^{\circledR}$ e Acapella ${ }^{\circledR}$;

4) Exercícios ventilatórios: respiração profunda, expiração profunda, prolongada, forçada ou lenta e o freno labial. A tosse e o huffing não foram categorizados, por se tratar, geralmente, da etapa final das terapias de remoção de secreção das vias aéreas, independente do método utilizado ${ }^{8}$.

\section{Resultados}

De um total de 1.526 artigos, 1.018 foram encontrados no PubMed, 501 no SciELO, três no LILACS, quatro na Cochrane e nenhum na base de dados PEDro. Desses, 50 foram excluídos, por estarem repetidos nas bases de dados utilizadas, e 1.454 pelo fato de não preencherem os critérios de elegibilidade desta revisão. Assim, foram incluídos, 22 estudos que utilizaram técnicas de FR, para a remoção de secreção das vias aéreas de crianças e adolescentes com FC. A Figura 1 apresenta o fluxograma em relação ao total de artigos encontrados.

Os artigos selecionados variaram do ano de 1980 a 2018, sendo que 12 (54,5\%) foram publicados posteriormente ao ano 2000. Quanto à origem dos estudos, nove (40,9\%) são do continente Oceânico, seguido da Europa (27,3\%) e América (27,3\%), entre outros. A maioria dos estudos $(54,5 \%)$ apresentou delineamento do tipo ensaio clínico randomizado. A amostra das pesquisas totalizou 708 participantes (701 com FC e sete saudáveis), com o tamanho amostral oscilando entre seis e 249 sujeitos, e a idade variando de três semanas a 18 anos de vida. Quanto às informações de função pulmonar (\% do previsto) disponíveis nos estudos, o $\mathrm{VEF}_{1}$ variou entre 43,8 e 97,5 e a CVF entre 54,5 e 100,7, evidenciando um leve/moderado comprometimento pulmonar. A Tabela 1 apresenta, detalhadamente, essas características. 
Figura 1 | Sistematização dos estudos incluídos nesta revisão.

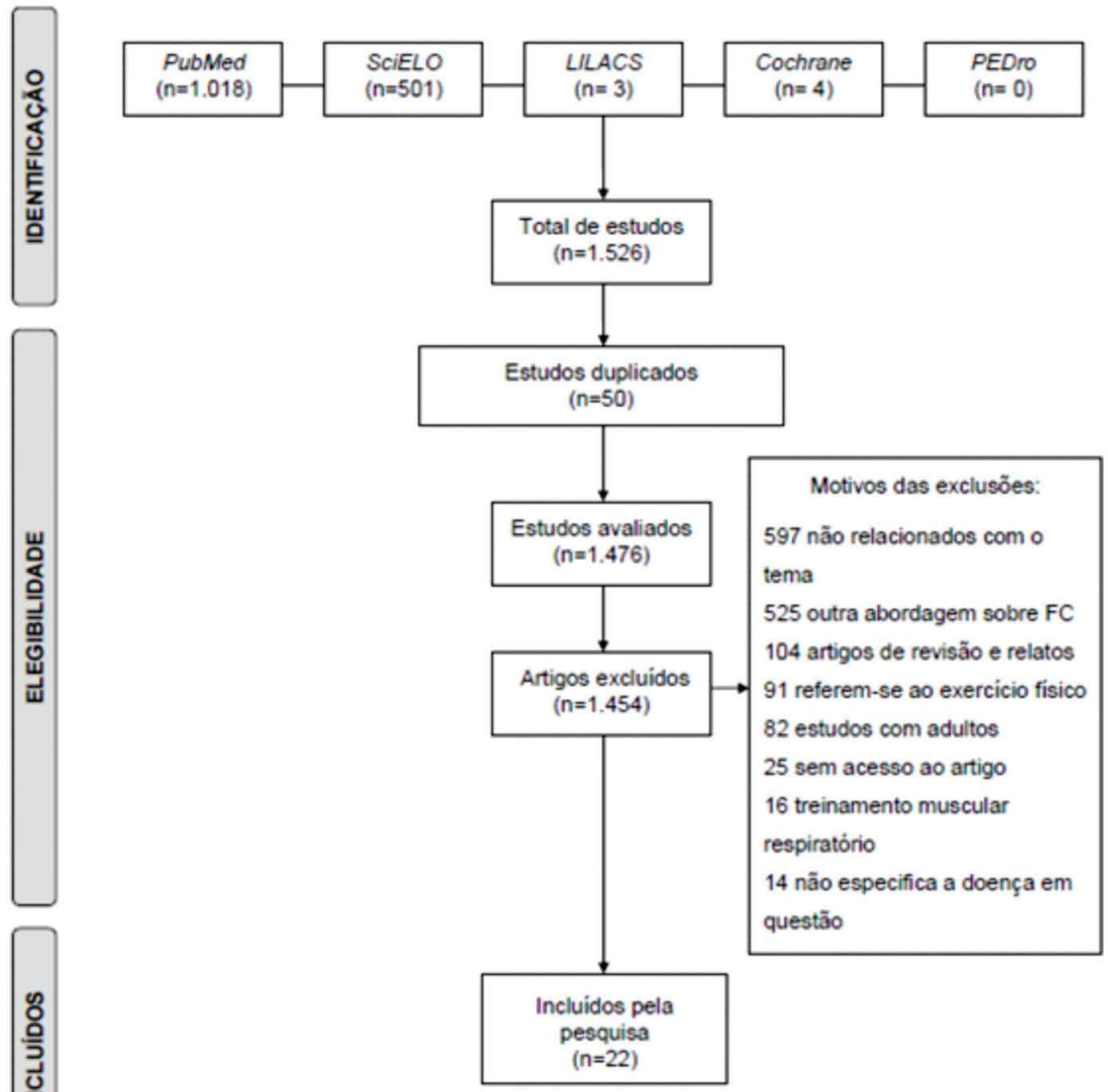




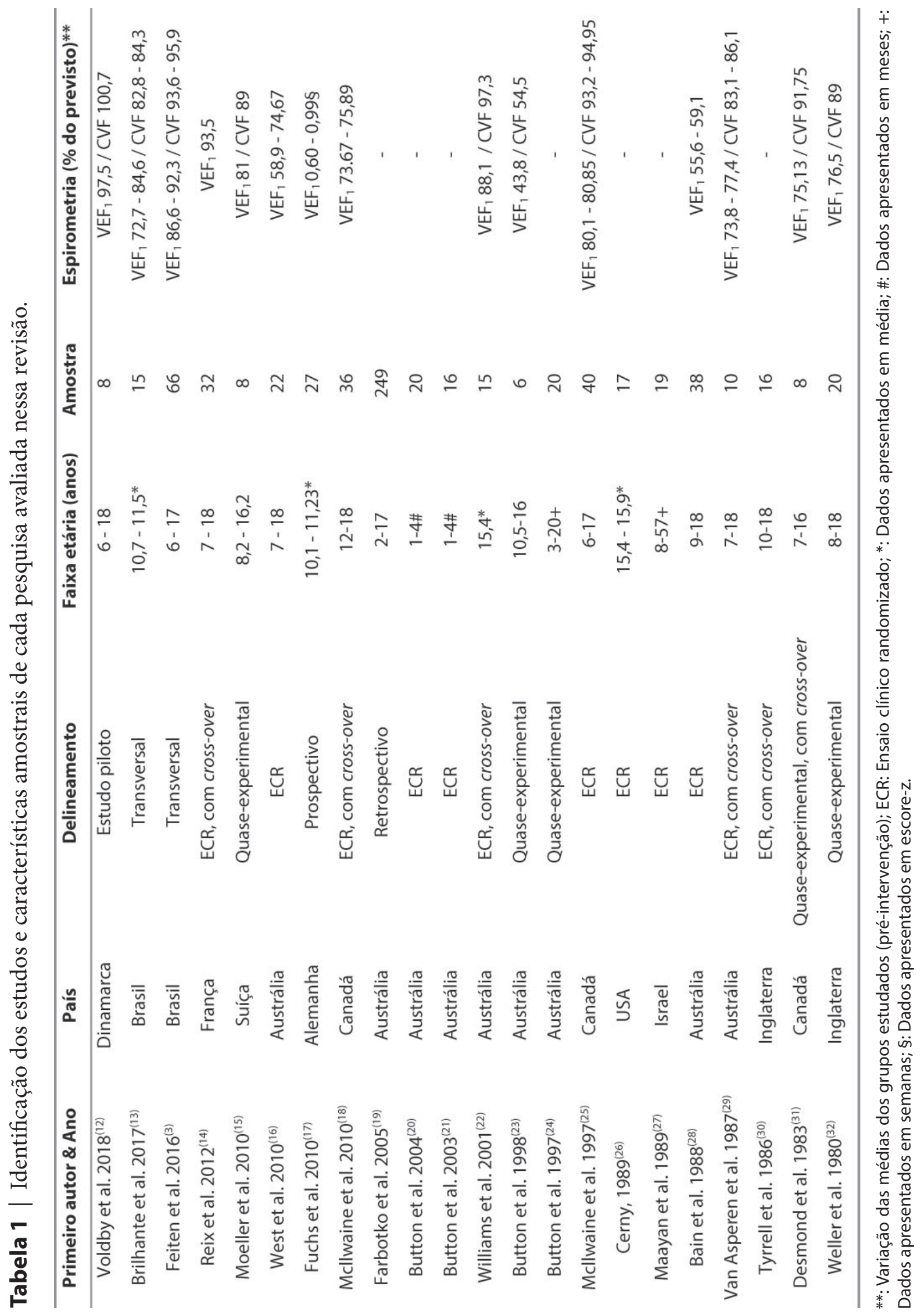

54 | ASSOBRAFIR Ciência. 2019 Abr;10(1):49-61 
A Tabela 2 demonstra a identificação das intervenções de fisioterapia utilizada nos estudos selecionados, para a remoção de secreção das vias aéreas de crianças e adolescentes com FC. Do total de 22 artigos localizados, 16/22 abordaram as intervenções convencionais, sendo, a drenagem postural, a percussão e a vibração, as mais frequentes. Nesses, cinco usaram somente as convencionais, enquanto 11 foram associadas com intervenções baseadas em volumes instrumentais ou exercícios ventilatórios. Ainda, 12/22 utilizaram intervenções instrumentais, sobressaindo-se o dispositivo/ máscara de PEP e o Flutter ${ }^{\circledast}$. Dessas, quatro usaram somente instrumentais, enquanto oito estavam associadas às demais terapêuticas. Quanto aos exercícios ventilatórios, eles foram estudados em 7/22 artigos, sendo avaliados sempre conjuntamente às outras intervenções reportadas. Já nas intervenções baseadas em volume (6/22 estudos), o ciclo ativo da respiração e a drenagem autogênica foram as mais frequentes. Neste caso, nenhum estudo investigou isoladamente essas intervenções de FR.

Quando se estratificaram os estudos em relação ao grupo etário, 4/22 incluíram somente lactentes ( $<2$ anos), no qual, todos utilizaram intervenções fisioterapêuticas convencionais, incluindo a drenagem postural, a percussão e a vibração. Nesses, 3/4 foram isolados de outras intervenções. Também, 17/22 englobaram indivíduos de seis a 18 anos, sendo que somente dois continham exclusivamente intervenções convencionais. Apenas 1/22 não pode ser classificado (lactente ou escolar/adolescente) devido à variação das idades.

Tabela 2 | Intervenções fisioterapêuticas utilizadas para a remoção de secreção das vias aéreas em crianças e adolescentes com FC nas pesquisas selecionadas.

\begin{tabular}{|c|c|c|}
\hline Primeiro autor \& Ano & Classificação das intervenções de FR & Descrição da intervenção \\
\hline Voldby et al. $2018^{(12)}$ & Instrumentais & $\begin{array}{l}\text { Tosse / Huffing } \\
\text { Dispositivo de PEP }\end{array}$ \\
\hline Brilhante et al. $2017^{(13)}$ & Instrumentais & Dispositivo de PEP \\
\hline Feiten et al. $2016^{(3)}$ & $\begin{array}{l}\text { - } \\
\text { Baseadas em volume } \\
\text { Instrumentais }\end{array}$ & $\begin{array}{l}\text { Tosse / Huffing } \\
\text { Expiração lenta total com a glote aberta } \\
\text { Máscara de PEP / PEP em selo d'água }\end{array}$ \\
\hline Reix et al. 2012 $2^{(14)}$ & $\begin{array}{l}\text { - } \\
\text { Convencionais } \\
\text { Baseadas em volume } \\
\text { Exercícios ventilatórios }\end{array}$ & $\begin{array}{l}\text { Huffing } \\
\text { TEMP } \\
\text { Ciclo ativo da respiração } \\
\text { Freno labial }\end{array}$ \\
\hline Moeller et al. $2010^{(15)}$ & $\begin{array}{l}\text { Baseadas em volume } \\
\text { Instrumentais }\end{array}$ & $\begin{array}{l}\text { Drenagem autogênica } \\
\text { Sistema de PEP / Flutter }\end{array}$ \\
\hline West et al. $2010^{(16)}$ & Instrumentais & Acappela® / Máscara de PEP \\
\hline Fuchs et al. $2010^{(17)}$ & Instrumentais & Flutter $^{\oplus}$ / Dispositivo de PEP \\
\hline Mcllwaine et al. $2010^{(18)}$ & $\begin{array}{l}\text { - } \\
\text { Convencionais } \\
\text { Baseadas em volume } \\
\text { Exercícios ventilatórios }\end{array}$ & $\begin{array}{l}\text { Huffing } \\
\text { Drenagem postural / Percussão / Vibração } \\
\text { Drenagem autogênica } \\
\text { Expiração profunda }\end{array}$ \\
\hline Farbotko et al. $2005^{(19)}$ & $\begin{array}{l}\text { Convencionais } \\
\text { Baseadas em volume } \\
\text { Instrumentais }\end{array}$ & $\begin{array}{l}\text { Drenagem postural } \\
\text { Drenagem autogênica } \\
\text { Dispositivo de PEP / Flutter }\end{array}$ \\
\hline Button et al. $2004^{(20)}$ & Convencionais & Drenagem postural / Percussão / Vibração \\
\hline Button et al. $2003^{(21)}$ & $\begin{array}{l}\text { Convencionais } \\
\text { Instrumentais }\end{array}$ & $\begin{array}{l}\text { Drenagem postural / Percussão / Vibração } \\
\text { Dispositivo de PEP }\end{array}$ \\
\hline Williams et al. $2001^{(22)}$ & $\begin{array}{l}\text { Convencionais } \\
\text { Baseadas em volume }\end{array}$ & $\begin{array}{l}\text { Percussão / Vibração } \\
\text { Ciclo ativo da respiração }\end{array}$ \\
\hline
\end{tabular}


Tabela 2 | Intervenções fisioterapêuticas utilizadas para a remoção de secreção das vias aéreas em crianças e adolescentes com FC nas pesquisas selecionadas.

\begin{tabular}{|c|c|c|}
\hline Primeiro autor \& Ano & Classificação das intervenções de FR & Descrição da intervenção \\
\hline Button et al. $1998^{(23)}$ & $\begin{array}{l}\text { Convencionais } \\
\text { Instrumentais } \\
\text { Exercícios ventilatórios }\end{array}$ & $\begin{array}{l}\text { Tosse } \\
\text { Drenagem postural / Percussão / Vibração } \\
\text { Máscara de PEP } \\
\text { Expiração forçada / Respiração profunda e lenta }\end{array}$ \\
\hline Button et al. $1997^{(24)}$ & Convencionais & Drenagem postural / Percussão / Vibração \\
\hline Mcllwaine et al. $1997^{(25)}$ & $\begin{array}{l}\text { Convencionais } \\
\text { Instrumentais } \\
\text { Exercícios ventilatórios }\end{array}$ & $\begin{array}{l}\text { Tosse } \\
\text { Drenagem postural / Percussão / Vibração } \\
\text { Máscara de PEP } \\
\text { Expiração forçada / Respiração profunda }\end{array}$ \\
\hline $\begin{array}{l}\text { Cerny, } 1989^{(26)} \\
\text { Maayan et al. } 1989^{(27)}\end{array}$ & $\begin{array}{l}\text { Convencionais } \\
\text { Convencionais }\end{array}$ & $\begin{array}{l}\text { Tosse } \\
\text { Drenagem postural / Percussão / Vibração } \\
\text { Drenagem postural / Percussão }\end{array}$ \\
\hline Bain et al. $1988^{(28)}$ & - & $\begin{array}{l}\text { Tosse / Huffing } \\
\text { Drenagem postural / Percussão / Vibração }\end{array}$ \\
\hline Van Asperen et al. $1987^{(29)}$ & $\begin{array}{l}\text { Convencionais } \\
\text { Instrumentais } \\
\text { Exercícios ventilatórios }\end{array}$ & $\begin{array}{l}\text { Tosse } \\
\text { Drenagem postural / Percussão } \\
\text { Máscara de PEP } \\
\text { Expiração forçada }\end{array}$ \\
\hline Tyrrell et al. $1986^{(30)}$ & $\begin{array}{l}\text { Convencionais } \\
\text { Instrumentais }\end{array}$ & $\begin{array}{l}\text { Tosse } \\
\text { Drenagem postural / Percussão } \\
\text { Máscara de PEP }\end{array}$ \\
\hline Desmond et al. $1983^{(31)}$ & $\begin{array}{l}\text { - } \\
\text { Convencionais } \\
\text { Exercícios ventilatórios }\end{array}$ & $\begin{array}{l}\text { Tosse } \\
\text { Percussão / Vibração } \\
\text { Respiracão profunda }\end{array}$ \\
\hline Weller et al. $1980^{(32)}$ & $\begin{array}{l}\text { Convencionais } \\
\text { Exercícios ventilatórios }\end{array}$ & $\begin{array}{l}\text { Tosse } \\
\text { Percussão / Vibração / Drenagem postural } \\
\text { Respiracão profunda / Expiração forcada }\end{array}$ \\
\hline
\end{tabular}

-: Sem classificação; FC: Fibrose cística; PEP: Pressão expiratória positiva; TEMP: Terapia expiratória manual passiva. FR: fisioterapia respiratória.

\section{Discussão}

$\mathrm{Na}$ presente revisão, foram identificados, 22 estudos que utilizaram intervenções fisioterapêuticas, para a remoção de secreção das vias aéreas de crianças e adolescentes com FC, com leve/moderado comprometimento pulmonar. Houve grande variabilidade nas intervenções reportadas pelas pesquisas, embora tenha ocorrido um leve predomínio das intervenções convencionais, nesse mapeamento conduzido pela revisão ${ }^{14,18-32}$. Dentre essas, as mais frequentes foram a drenagem postural, a percussão e a vibração, sendo que muitas estavam associadas às demais terapêuticas ${ }^{14,18,19,21-23,25,29-32}$.

$\mathrm{O}$ guia de tratamento da $\mathrm{FC}^{10}$ relata que, apesar dos efeitos da drenagem postural serem questionáveis, sua aplicação de forma combinada a outras intervenções de FR parece ser eficaz. Isso corrobora com os achados deste estudo, no qual, a drenagem postural esteve associada com outras intervenções ${ }^{18-21,23-30,32}$, incluindo a vibração e/ou a percussão $0^{18,20,21,23-30,32}$. Também, é relatado que a percussão traz alguns efeitos adversos, como microatelectasia, broncoespasmo e queda da saturação de oxigênio, o que torna sua aplicação controversa em determinados casos10. Nesta revisão, embora a percussão não tenha sido utilizada, isoladamente, em estudo algum ${ }^{18,20,21-32}$, uma pequena parcela 
reportou refluxo gastroesofágico, queda da saturação de oxigênio e o aumento de complicações respiratórias ${ }^{20,21,23,24}$. Apesar disso, essas terapêuticas são indicadas ${ }^{10}$ e a percussão foi uma das intervenções convencionais mais frequentes nos estudos selecionados. Segundo Main et al. ${ }^{33}$, a ausência de diferença entre os tratamentos pode suportar sua recomendação, já que não há distinções, quanto à eficácia entre as intervenções fisioterapêuticas. No entanto, considerando os relatos adversos documentados ${ }^{10,20,21,23,24}$, a aplicação da percussão deve ser executada com cautela, sendo necessário avaliar e modificar a terapia, na ocorrência dessas complicações, durante o atendimento.

Sabe-se que a escolha das intervenções de FR é influenciada pela idade, gravidade clínica, avaliação e resposta terapêutica. No entanto, esta revisão não pode investigar a influência de todos esses fatores, considerando que apenas a idade e a gravidade clínica (função pulmonar) estiveram

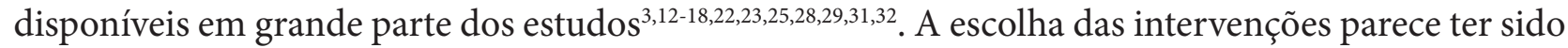
influenciada pela faixa etária nos estudos selecionados, já que, conforme há o aumento da idade, ocorre a redução por terapias exclusivamente convencionais ${ }^{14,18,19,22,23,25,29-32}$. Isso sugere que quanto maior a idade dos pacientes, maiores são a autonomia e a chance do uso de intervenções fisioterapêuticas independentes. Além do mais, como a gravidade dos participantes, avaliada pela espirometria, foi considerada leve a moderada, poderia ser aplicada qualquer terapêutica para a remoção de secreção das vias aéreas, fato esse evidenciado pela variabilidade das intervenções utilizadas.

A escolha por terapias menos independentes, como no caso das intervenções fisioterapêuticas convencionais, deve ser, minuciosamente, avaliada, principalmente, em amostras a partir da adolescência, devido à possibilidade de menor adesão à prática da FR. Sugere-se a utilização dessas terapias, em amostras mais jovens, considerando tanto relação terapeuta dependente quanto o fato de que todos os estudos selecionados em lactentes utilizaram intervenções convencionais ${ }^{20,21,24,27}$. Embora esta revisão tenha selecionado um número restrito de trabalhos em lactentes ${ }^{20,21,24,27}$, parece que a escolha por intervenções exclusivamente convencionais foi quase unânime nessas amostras ${ }^{20,24,27}$. Nos estudos selecionados, não foram localizadas pesquisas que abordassem outros manejos, incluindo a expiração lenta e prolongada e a aceleração do fluxo expiratório. Assim, recomenda-se que futuros estudos comparem a efetividade entre essas intervenções, objetivando avaliar se existe superioridade em alguma delas.

A diretriz brasileira de $\mathrm{FC}^{4}$ sugere que a independência do paciente é um fator imprescindível para a escolha da intervenção fisioterapêutica a ser utilizada, conferindo uma preferência pelo uso de instrumentos como os dispositivos/máscara de PEP, Flutter ${ }^{\circledR}$, Shaker ${ }^{\circledR}$ e Acapella $^{\circledR}$. Recentemente, um estudo realizado ${ }^{8}$ em diversos centros de referência da doença, no Brasil, evidenciou uma variabilidade na recomendação das intervenções de FR, embora as mais utilizadas foram os dispositivos de oscilação oral de alta frequência $\left(\right.$ Flutter $^{\circledR}$, Shaker ${ }^{\circledR}$ e Acapella ${ }^{\circledR}$ ) e as de PEP, seguidos das intervenções baseadas em volume. Nos resultados desta pesquisa, as intervenções instrumentais ficaram em segundo lugar ${ }^{3,12,13,15-17,19,21,23,25,29,30}$, sendo elas isoladas ou associadas às terapias, sobressaindo-se os dispositivos/máscara de PEP e o Flutter ${ }^{\circledR}$. Estas intervenções geram independência tanto aos pacientes quanto à família, sendo que sua escolha, no público jovem, pode acarretar uma melhor adesão ao tratamento ${ }^{34,35}$, visto que a FR faz parte, diariamente, da vida do indivíduo com $\mathrm{FC}^{4}$. Além disso, uma baixa adesão às recomendações da fisioterapia está associada com piores achados radiológicos, maior número de hospitalizações e diminuição da qualidade de vida, em crianças e adolescentes com $\mathrm{FC}^{3}$.

Nesta revisão, 7/22 pesquisas utilizaram exercícios ventilatórios ${ }^{14,18,23,25,29,31,32}$, como a respiração 
profunda e os mais variados tipos de expiração, além das intervenções baseadas em volume, em 6/22 estudos ${ }^{3,14,15,18,19,22}$, incluindo o ciclo ativo da respiração e a drenagem autogênica. Como esperado, a escolha destas ocorreu em amostras, nas quais, já se pressupõe independência ${ }^{3,14,15,18,19,22,23,25,29,31,32}$, visto que necessitam de autonomia para a realização. Isso pode ser atribuído ao maior entendimento necessário para a execução dessas terapias, considerando que envolvem diferentes volumes e/ou manobras respiratórias. Suas execuções podem ser outras possibilidades para a remoção de secreção das vias aéreas, em amostras pediátricas mais velhas, já que esses pacientes realizam a prática diária de FR, ao longo da vida, podendo modificá-las, de acordo com sua preferência. Independente da intervenção de escolha, a prática de FR previne complicações respiratórias, otimiza a função pulmonar e promove melhor treinamento físico ${ }^{7,10}$. Cabe, ao fisioterapeuta, assessorar toda família, quanto aos cuidados domiciliares, à terapia inalatória e às tecnologias para manutenção do quadro respiratório ${ }^{3,10}$.

Como perspectivas, não se recomenda, o uso generalizado e rotineiro dessas intervenções, para essa população, baseado na reflexão terapêutica dos achados encontrados. Cabe ao fisioterapeuta, em conjunto com o paciente e/ou sua família, escolher, testar e evoluir condutas de FR, de acordo com a peculiaridade, a idade, a gravidade, a preferência e a resposta terapêutica, não havendo uma intervenção fisioterapêutica considerada padrão-ouro e/ou que convenha usar, desde o nascimento até por toda sua vida.

A presente revisão encontrou como limitações não verificar, nos estudos incluídos, cada intervenção fisioterapêutica, isoladamente, para procurar identificar a mais eficaz. Porém, a diretriz mais recente $e^{4}$ conta com recomendações acerca das condutas que seriam mais indicadas, e o guia de prática clínica do ano passado ${ }^{10}$ traz evidências acerca do tratamento com a FR. Sem contar que revisões, que usaram bases de dados diferentes, tanto em 2013 como em $2019^{33,36}$, verificaram não ser possível constatar se uma intervenção é superior à outra. Além do mais, a ausência de alguns descritores na estratégia de busca, mesmo que não controlados, por exemplo, "airway clearance techniques" ou "chest physiotherapy", podem ter reduzido o número de estudos identificados sobre o tema.

\section{Conclusão}

Os resultadosdesta revisão evidenciaram grande variabilidadenasintervenções fisioterapêuticas, para a remoção de secreção das vias aéreas neste mapeamento realizado em crianças e adolescentes com FC. No entanto, houve um pequeno predomínio de intervenções convencionais, sendo, a drenagem postural, a vibração e a percussão, as mais executadas. Além disso, parece que a escolha das intervenções de FR, utilizadas pelas pesquisas, foi influenciada pela faixa etária, considerando que, conforme há aumento da idade, ocorre redução por manejos, exclusivamente, convencionais.

\section{Referências}

1. Paranjape SM, Mogayzel PJ Jr. Cystic Fibrosis. Pediatr Rev. 2014 May;35(5):194-205. doi: 10.1542/ pir.35-5-194.

2. Rafeeq MM, Murad HAS. Cystic fbrosis: current therapeutic targets and future approaches. J Transl Med. 2017 Apr 27;15(1):84. doi: 10.1186/s12967-017-1193-9.

3. Feiten Tdos S, Flores JS, Farias BL, Rovedder PM, Camargo EG, Dalcin Pde T, et al. Respiratory 
therapy: a problem among children and adolescents with cystic fibrosis. J Bras Pneumol. 2016 JanFeb;42(1):29-34. doi: 10.1590/S1806-37562016000000068.

4. Athanazio RA, Silva Filho LVRF, Vergara AA, Ribeiro AF, Riedi CA, Procianoy EFA, et al. Diretrizes brasileiras de diagnóstico e tratamento da fibrose cística. J Bras Pneumol. 2017;43(3):219-45. doi: 10.1590/S1806-37562017000000065.

5. Dalcin PDTR, Abreu e Silva FAD. Cystic fibrosis in adults: diagnostic and therapeutic aspects. J Bras Pneumol. 2008 Feb;34(2):107-17.

6. Sociedade Brasileira de Pneumologia. Fibrose Cística: Diagnóstico e tratamento [internet]. Brasília: SBPT; [2019; cited 2019 Mar 21]. Available from: https://sbpt.org.br/portal/publico-geral/fibrosecistica-diagnostico-tratamento/

7. Lannefors L, Button BM, McIlwaine M. Physiotherapy in infants and young children with cystic fibrosis: current practice and future developments. J R Soc Med. 2004;97 Suppl 44(Suppl 44):8-25.

8. Donadio MV, Campos NE, Vendrusculo FM, Stofella AM, Almeida ACS, Ziegler B, et al. Respiratory physical therapy techniques recommended for patients with cystic fibrosis treated in specialized centers. Braz J Phys Ther. 2019 Nov 29:S1413-3555(19)30062-0. doi: 10.1016/j.bjpt.2019.11.003. Online ahead of print.

9. Warnock L, Gates A. Chest physiotherapy compared to no chest physiotherapy for cystic fibrosis. Cochrane Database Syst Rev. 2015 Dec 21;2015(12):CD001401. doi: 10.1002/14651858.CD001401. pub3.

10. Schivinski CIS, Stofella AM, Almeida ACS, Scalco JC, Parazzi PLF, Gonçalves Wamosy RMG. Parte 1. Avaliação do paciente com fibrose cística [Recomendação Brasileira de Fisioterapia na Fibrose Cística, um guia de boas práticas clínicas]. ASSOBRAFIR Ciência. 2019 Maio;10(Supl 1):2160 .

11. Brasil. Lei federal no 8069, de 13 de julho de 1990. Estatuto da criança e do adolescente [Internet]. Brasília; 1990 [cited 2019 Mar 21]. Available from: https://legislacao.presidencia.gov.br/atos/?tipo=L EI\&numero $=8069 \& a n o=1990 \&$ ato $=461 \mathrm{cXRq} 1 \mathrm{keFpWT13a}$

12. Voldby C, Green K, Rosthøj S, Kongstad T, Philipsen L, Buchvald F, et al. The effect of time-of-day and chest physiotherapy on multiple breath washout measures in children with clinically stable cystic fibrosis. PLoS One. 2018 Jan 10;13(1):e0190894. doi: 10.1371/journal.pone.0190894. eCollection 2018.

13. Brilhante SA, Florêncio RB, Gualdi LP, Resqueti VR, Aliverti A, Andrade AFD, et al. Comparison of different levels of positive expiratory pressure on chest wall volumes in healthy children and patients with fibrosis. Fisioter Pesqui. 2017 Jul-Sept;24(3):311-20. doi: 10.1590/1809-2950/17015824032017

14. Reix P, Aubert F, Werck-Gallois MC, Toutain A, Mazzocchi C, Moreux N, et al. Exercise with incorporated expiratory manoeuvres was as effective as breathing techniques for airway clearance in children with cystic fibrosis: a randomised crossover trial. J Physiother. 2012;58(4):241-7. doi: 10.1016/S1836-9553(12)70125-X.

15. Moeller A, Stampfli SF, Rueckert B, Rechsteiner T, Hamacher J, Wildhaber JH. Effects of a ShortTerm Rehabilitation Program on Airway Inflammation in Children With Cystic Fibrosis. Pediatr Pulmonol. 2010 Jun;45(6):541-51. doi: 10.1002/ppul.21167. 
16. West K, Wallen M, Follett J. Acapella vs. PEP mask therapy: A randomised trial in children with cystic fibrosis during respiratory exacerbation. Physiother Theory Pract. 2010 Apr 22;26(3):143-9. doi: 10.3109/09593980903015268.

17. Fuchs SI, Toussaint S, Edlhaimb B, Ballmann M, Gappa M. Short-Term Effect of Physiotherapy on Variability of the Lung Clearance Index in Children With Cystic Fibrosis. Pediatr Pulmonol. 2010 Mar;45(3):301-6. doi: 10.1002/ppul.21180.

18. McIlwaine M, Wong LT, Chilvers M, Davidson GF. Long-Term Comparative Trial of Two Different Physiotherapy Techniques; Postural Drainage With Percussion and Autogenic Drainage, in the treatment of Cystic Fibrosis. Pediatr Pulmonol. 2010 Nov;45(11):1064-9. doi: 10.1002/ppul.21247.

19. Farbotko K, Wilson C, Watter P, MacDonald J. Change in physiotherapy management of children with cystic fibrosis in a large urban hospital. Physiother Theory Pract. Jan-Mar 2005;21(1):13-21. doi: 10.1080/09593980590911480.

20. Button BM, Heine RG, Catto-Smith AG, Phelan PD, Olinsky A. Chest physiotherapy, gastrooesophageal reflux, and arousal in infants with cystic fibrosis. Arch Dis Child. 2004 May;89(5):435-9. doi: 10.1136/adc.2003.033100.

21. Button BM, Heine RG, Catto-Smith AG, Olinsky A, Phelan PD, Ditchfield MR, et al. Chest Physiotherapy in Infants With Cystic Fibrosis: To Tip or Not? A Five-Year Study. Pediatr Pulmonol. 2003 Mar;35(3):208-13. doi: 10.1002/ppul.10227.

22. Williams MT, Parsons DW, Frick RA, Ellis ER, Martin AJ, Giles SE, et al. Acute respiratory infection in patients with cystic fibrosis with mild pulmonary impairment: Comparison of two physiotherapy regimens. Aust J Physiother. 2001;47(4):227-36. doi: 10.1016/s0004-9514(14)60270-1.

23. Button BM, Heine RG, Catto-Smith AG, Phelan PD. Postural drainage in cystic fibrosis: Is there a link with gastro-oesophageal reflux? J Paediatr Child Health. 1998 Aug;34(4):330-4. doi: 10.1046/j.1440-1754.1998.00236.x.

24. Button BM, Heine RG, Catto-Smith AG, Phelan PD, Olinsky A. Postural drainage and gastrooesophageal reflux in infants with cystic fibrosis. Arch Dis Child. 1997 Feb;76(2):148-50. doi: 10.1136/ adc.76.2.148.

25. Mcllwaine M, Wong LT, Peacock D, Davidson GF. Long-term comparative trial of conventional postural drainage and percussion versus positive expiratory physiotherapy in the treatment of cystic fibrosis. J Pediatr. 1997 Oct;131(4):570-4. doi: 10.1016/s0022-3476(97)70064-7.

26. Cerny FJ. Relative Effects of Bronchial Drainage and Exercise for In-Hospital Care of Patients with Cystic Fibrosis. Phys Ther. 1989 Aug;69(8):633-9. doi: 10.1093/ptj/69.8.633.

27. Maayan C, Bar-Yishay E, Yaacobi T, Marcus Y, Katznelson D, Yahav Y, et al. Immediate Effect of Various Treatments on Lung Function in Infants with Cystic Fibrosis. Respiration. 1989;55(3):14451. doi: $10.1159 / 000195725$.

28. Bain J, Bishop J, Olinsky A. Evaluation of directed coughing in cystic fibrosis. Br J Dis Chest. 1988 Apr;82(2):138-48. doi: 10.1016/0007-0971(88)90034-4.

29. Van Asperen PP, Jackson L, Hennessy P, Brown J. Comparison of a positive expiratory pressure (PEP) mask with postural drainage in patients with cystic fibrosis. Aust Paediatr J. 1987 Oct;23(5):283- 
4. doi: 10.1111/j.1440-1754.1987.tb00272.x.

30. Tyrrell JC, Hiller EJ, Martin J. Face mask physiotherapy in cystic fibrosis. Arch Dis Child. 1986 Jun;61(6):598-600. doi: 10.1136/adc.61.6.598.

31. Desmond KJ, Schwenk WF, Thomas E, Beaudry PH, Coates AL. Immediate and long-term physiotherapy in patients effects of chest with cystic fibrosis. J Pediatr. 1983 Oct;103(4):538-42. doi: 10.1016/s0022-3476(83)80579-4.

32. Weller PH, Bush E, Preece MA, Matthew DJ. Short-Term Effects of Chest Physiotherapy on Pulmonary Function in Children with Cystic Fibrosis. Respiration. 1980;40(1):53-6. doi: $10.1159 / 000194251$.

33. Main E, Prasad A, Schans C. Conventional chest physiotherapy compared to other airway clearance techniques for cystic fibrosis. Cochrane Database Syst Rev. 2005 Jan 25;2005(1):CD002011. doi: 10.1002/14651858.CD002011.pub2.

34. McIlwaine M, Button B, Dwan K. Positive expiratory pressure physiotherapy for airway clearance in people with cystic fibrosis. Cochrane Database Syst Rev. 2015 Jun 17;(6):CD003147. doi: 10.1002/14651858.CD003147.pub4.

35. Morrison L, Innes S. Oscillating devices for airway clearance in people with cystic fibrosis. Cochrane Database Syst Rev. 2017 May 4;5(5):CD006842. doi: 10.1002/14651858.CD006842.pub4.

36. Wilson LM, Morrison L, Robinson KA. Airway clearance techniques for cystic fibrosis: an overview of Cochrane systematic reviews. Cochrane Database Syst Rev. 2019 Jan 24;1(1):CD011231. doi: 10.1002/14651858.CD011231.pub2.

Submissão em: 02/09/2019

Aceito em: 04/06/2020 